\title{
Central Nervous System Histoplasma-Associated Post-infectious Inflammatory Response Syndrome (Histo-PIIRS)
}

\author{
Owen Dean ${ }^{1} \cdot$ Seher Anjum ${ }^{1} \cdot$ Bryan Hess $^{2}$ - Dima A. Hammoud ${ }^{3} \cdot$ Deena Athas $^{4}$ - Joseph Wheat ${ }^{5}$. \\ Peter R. Williamson ${ }^{1}$
}

Received: 22 May 2020 / Accepted: 22 December 2020 / Published online: 2 January 2021

(C) This is a U.S. government work and not under copyright protection in the U.S.; foreign copyright protection may apply 2021

\begin{abstract}
We present a case of central nervous system (CNS) histoplasmosis in a previously healthy adult with hepatitis C (HCV) presenting with neurological symptoms refractory to antifungal therapy and ventriculoperitoneal (VP) shunting 4 months after initial diagnosis. Persistent symptoms were thought to be inflammatory rather than infectious given negative cerebrospinal fluid (CSF) and serum fungal antigens. The patient promptly improved after initiation of corticosteroid therapy. Elevated CSF cytokines and regional enhancement on brain MRI resolved with corticosteroid treatment. This is the first case of Histoplasma-associated post-infectious inflammatory response syndrome (Histo-PIIRS) documented by CSF cytokine reduction in response to corticosteroid therapy.
\end{abstract}

Keywords Fungal meningitis $\cdot$ histoplasma $\cdot$ inflammation $\cdot$ central nervous system

\section{Introduction}

Histoplasmosis is caused by the inhalation of the dimorphic fungus Histoplasma capsulatum var. capsulatum or duboisii. Although infection is generally asymptomatic or mild, Histoplasma can cause severe pulmonary and disseminated disease, particularly in immunocompromised populations [1]. CNS disease occurs in $5-10 \%$ of patients with disseminated infection and has a 1-year mortality of 20-25\% despite therapy. A small proportion of patients with CNS histoplasmosis are

Peter R. Williamson

williamsonpr@mail.nih.gov

1 Laboratory of Clinical Immunology and Microbiology, National Institute of Allergy and Infectious Diseases, National Institutes of Health, Bld 10, Rm 11C208, 9000 Rockville Pike, Bethesda, MD 20892, USA

2 Division of Infectious Diseases, Sidney Kimmel Medical College at Thomas Jefferson University, Philadelphia, PA, USA

3 Center for Infectious Disease Imaging, Radiology and Imaging Sciences, Clinical Center, National Institutes of Health, Bethesda, MD, USA

4 Division of Infectious Diseases, Gundersen Health System, La Crosse, WI, USA

5 MiraVista Diagnostics, Indianapolis, IN, USA previously healthy prior to infection [2]. Immune reconstitution inflammatory syndrome (IRIS) has been reported in association with histoplasmosis in HIV-infected patients [3], and more recently, in those on waning TNF-alpha inhibition [4]. Here, we present the first report of an IRIS-like, PIIRS in a non-HIV patient with history of $\mathrm{HCV}$ infection and CNS histoplasmosis, based on elevated CSF cytokines in the setting of improved serologic and antigenic fungal parameters.

\section{Case}

A 23-year-old male with a history of intravenous heroin use began experiencing diffuse headaches in the absence of significant fever. After 4 months of symptoms and presenting to the emergency department multiple times, he was admitted due to concerns of meningitis. His initial lumbar puncture revealed a lymphocytic pleocytosis $\left(353 \mathrm{cells} / \mathrm{mm}^{3}, 65 \%\right.$ lymphocytes, $36 \%$ segmented neutrophils), low glucose $8 \mathrm{mg} / \mathrm{dL}$, high protein $234 \mathrm{mg} / \mathrm{dL}$, and no organisms on gram stain (Fig. 1). He was diagnosed with CNS histoplasmosis by serum and cerebrospinal fluid (CSF) enzyme immunoassay (EIA) antibody testing. At that time (day 0 ), his serum $(1,3)-\beta$-D-glucan was $>500 \mathrm{pg} / \mathrm{mL}$ (normal $<20 \mathrm{pg} / \mathrm{mL}$ ) and $<31 \mathrm{pg} / \mathrm{mL}$ (normal $<31 \mathrm{pg} / \mathrm{mL}$ ) in the CSF (Table 1). Alternative diagnoses including coccidioidomycosis, Lyme disease, HTLV, 
Table 1 Diagnostic studies in CNS histoplasmosis

\begin{tabular}{|c|c|c|c|c|c|c|c|c|c|c|c|c|}
\hline & \multicolumn{2}{|c|}{ Parameters $[5,6,27]$} & \multicolumn{10}{|c|}{ Results, days after diagnosis } \\
\hline & Sensitivity (\%) & Specificity (\%) & 0 & 114 & 117 & 119 & 122 & 126 & 161 & 183 & 217 & 658 \\
\hline \multicolumn{13}{|l|}{ CSF antibody EIA } \\
\hline IgG & 82.2 & 94.8 & $80^{\mathrm{a}}$ & & & & & & & & & \\
\hline $\operatorname{IgM}$ & 31.1 & 97.4 & $21.6^{\mathrm{a}}$ & & & & & & & & & \\
\hline CSF antigen EIA & 78.0 & 96.6 & & & Neg. ${ }^{a}$ & & & & & & & \\
\hline CSF beta-D-glucan $(\mathrm{pg} / \mathrm{mL})^{*}$ & 53.2 & 86.9 & $<31^{\mathrm{a}}$ & & & & & & & & & \\
\hline CSF antibody by $\mathrm{CF}^{* *}$ & 50.0 & 92.9 & & & & & & & & & & \\
\hline Yeast phase & ND & ND & & & $\geq 1: 16$ & $<1: 8$ & $1: 4$ & & $1: 8$ & $1: 8$ & $<1: 1$ & $<1: 1$ \\
\hline Mycelial phase & ND & ND & & & $<1: 1$ & $<1: 8$ & $1: 1$ & & $<1: 1$ & $<1: 1$ & $<1: 1$ & $<1: 1$ \\
\hline CSF fungal detection by PCR & ND & ND & & & & Neg. ${ }^{\mathrm{c}}$ & & & & & & \\
\hline CSF culture & 19.1 & 100.0 & & Neg. ${ }^{a}$ & & & & & & & & \\
\hline \multicolumn{13}{|l|}{ Serum antibody EIA } \\
\hline IgG & ND & ND & $80^{\mathrm{a}}$ & & & & & & & & & \\
\hline $\operatorname{IgM}$ & ND & ND & Neg. ${ }^{\text {a }}$ & & & & & & & & & \\
\hline Serum antigen EIA & 77 & ND & Neg. ${ }^{\text {a }}$ & Neg. ${ }^{a}$ & & & & Neg. ${ }^{\text {a }}$ & & & & \\
\hline Serum beta-D-glucan $(\mathrm{pg} / \mathrm{mL})$ & ND & ND & $>500^{\mathrm{a}}$ & & & & & & & & & \\
\hline Urine antigen EIA & 77 & ND & Neg. ${ }^{a}$ & & & & & $<0.5 \mathrm{ng}^{\mathrm{b}}$ & & & & \\
\hline
\end{tabular}

$E I A$ enzyme immunoassay, $C F$ complement fixation, $P C R$ polymerase chain reaction, $N e g$ negative, $N D$ no data

*Federal Drug Administration approved cutoff for CSF $(1,3)$ beta-D-glucan is $31 \mathrm{pg} / \mathrm{mL}$

**Detection of antibody by CF in undiluted CSF is considered positive

${ }^{\text {a }}$ MiraVista Diagnostics

${ }^{\mathrm{b}}$ Quest Diagnostics

${ }^{\mathrm{c}}$ University of Washington Molecular Diagnostics Microbiology Section

HSV, syphilis, brucellosis, leptospirosis, toxoplasmosis, and Legionnaire's disease were ruled out by relevant serologies. TB exposure was excluded by a negative quantiferon test and results of a meningitis/encephalitis CSF panel were negative. His serum CD4+ T cell count was 609 cells $/ \mathrm{mm}^{3}$ and his Creactive protein (CRP) was $7.11 \mathrm{mg} / \mathrm{dL}$. Immune workup including HIV-1 antibody, HIV-1 antigen, and HIV-2 antibody, Hepatitis A and B antibody were non-reactive (day 0). Although his initial HCV antibody was negative (day 0), it was reactive on day 14 and his viral load was 1,520,000 IU/L. He was treated with liposomal amphotericin for 4 weeks and transitioned to an 11-month itraconazole course on discharge. He was started on sofosbuvir and velpatasvir for HCV management due to high viral loads and liver enzymes. He was from Pennsylvania and worked in heating, ventilation, and air conditioning. He had a dog, but denied any exposure to rodents or birds.

Four months later, he was readmitted with severe headache, fevers, meningismus, and difficulty walking. An MRI of the brain revealed enhancement of the right internal auditory canal and cochlea concerning for a meningeal process (day 110). CRP had declined to $4.10 \mathrm{mg} / \mathrm{dL}$ and the patient had a low fever $\left(T_{\max } 100.3 \mathrm{~F}\right)$. A lumbar puncture (day 113 ) demonstrated low glucose and pleocytosis (Fig. 1a, c). Recurrence of CNS histoplasmosis was suspected given a positive CSF antibody by complement fixation (CF) on repeat lumbar puncture, despite other Histoplasma testing, including CSF fungal PCR and antigen by EIA, remaining negative (Table 1). Itraconazole was replaced with liposomal amphotericin B because of the suspected relapse.

On day 112, his HCV viral load had declined to 168,000 IU/L with improved aspartate transaminase (AST) and alanine aminotransferase (ALT) $57 \mathrm{U} / \mathrm{L}$ and $37 \mathrm{U} / \mathrm{L}$, respectively. HIV-1/2 status was also re-evaluated with a 4 th generation antibody test and was negative.

Two weeks later (day 123), a brain MRI revealed abnormal enhancement along the surface of the brainstem (interpeduncular cistern and surface of the pons and medulla) and multiple cranial nerves, including the internal auditory canals consistent with granulomatous meningitis with involvement of vestibular anatomy on dedicated studies of the internal auditory canal (Fig. 2a-d). There was also focal solid enhancement in the prepontine cistern, suspicious for a granuloma or early abscess. An LP on day 137 revealed pleocytosis $\left(5.3 \times 10^{4}\right.$ leukocytes $/ \mu \mathrm{L}, 63 \%$ lymphocytes, $27 \%$ segmented neutrophils), low glucose (32 mg/dL), and 

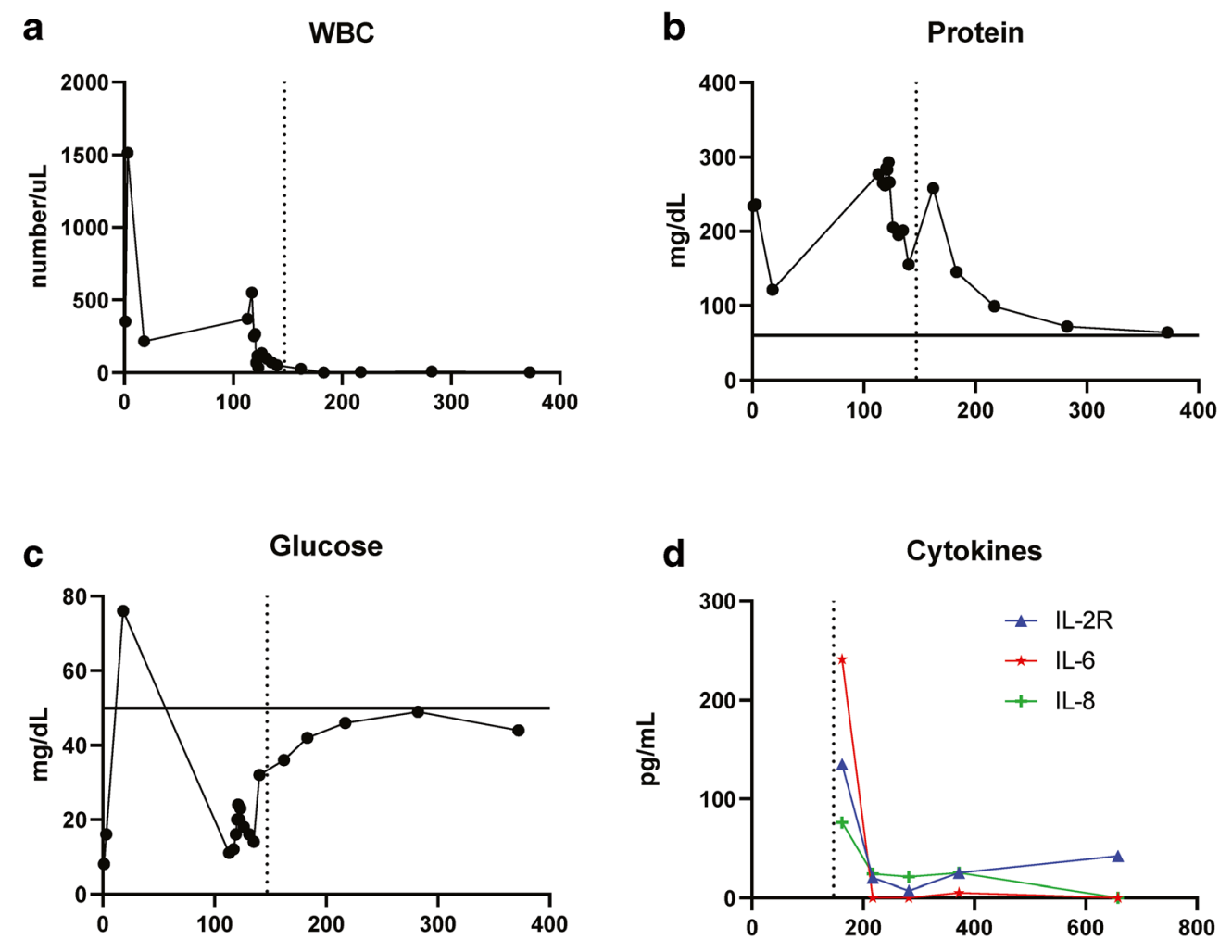

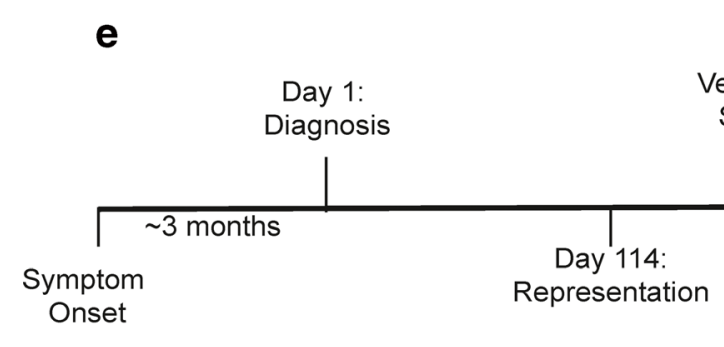

Fig. 1 Longitudinal cerebrospinal fluid findings and case timeline. a-d Indicated CSF parameters. White blood count (WBC). The vertical dotted line represents the start of prednisone therapy. The solid horizontal lines represent normal values (CSF glucose $>50 \mathrm{mg} / \mathrm{dL}$, CSF protein $<80 \mathrm{mg}$ ) $\mathrm{dL}, \mathrm{WBC}<5 / \mu \mathrm{L})$. Tumor necrosis factor-alpha, IL-2, IL-12, IL-4, IL-5,

high protein $(155 \mathrm{mg} / \mathrm{dL})$. A VP shunt was placed due to worsening headaches and persistently elevated intracranial pressure (day 141). Following surgery, the patient's headaches briefly improved but subsequently worsened. No shunt malfunction was detected at the time.

Prednisone $40 \mathrm{mg}$ daily was initiated on day 147 because of worsening clinical condition and the suspicion of an inflammatory syndrome. Two days later his headaches dissipated, his gait improved, and he was discharged to a nursing facility. CSF cytokines measured 2 days following corticosteroid initiation revealed elevated IL-6, soluble CD25, and IL-8 levels (Fig. 1d). Other autoimmune workup consisted of a negative rheumatoid factor, anti-nuclear antibody, antineutrophil
Day 141:

Ventriculoperitoneal Shunt Placement Day 150:

Discharge

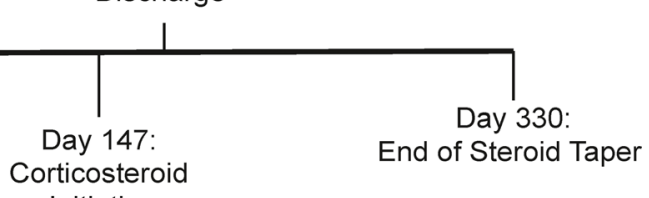

IL-5, IL-10, IL-13, IL-17, and IL-1- $\beta$ were not significantly elevated in the CSF at any time point and therefore not shown. CSF cytokines obtained by ARUP Laboratories. WBC: white blood cell count. Soluble CD25 is also known as IL-2 receptor

cytoplasmic antibody (ANCA) panel, anti-cyclic citrullinated peptide ab, anti-mitochondrial antibody, anti-smooth muscle $\mathrm{Ab}$, and negative tissue transglutaminase $\mathrm{IgA} / \mathrm{IgG}$. He was transitioned to itraconazole $200 \mathrm{mg}$ TID following the second course of 4 weeks of amphotericin B. A brain MRI on day 201 showed resolution of previous abnormal enhancement along the brainstem, internal auditory canals, and prepontine cistern (Fig. 2e).

His hospital course was complicated by recurrent knee arthritis. During his first admission, he was diagnosed with bilateral methicillin-sensitive Staphylococcal aureus (MSSA) septic arthritis treated with intravenous cefazolin and irrigation. Knee pain and swelling persisted through readmission. 


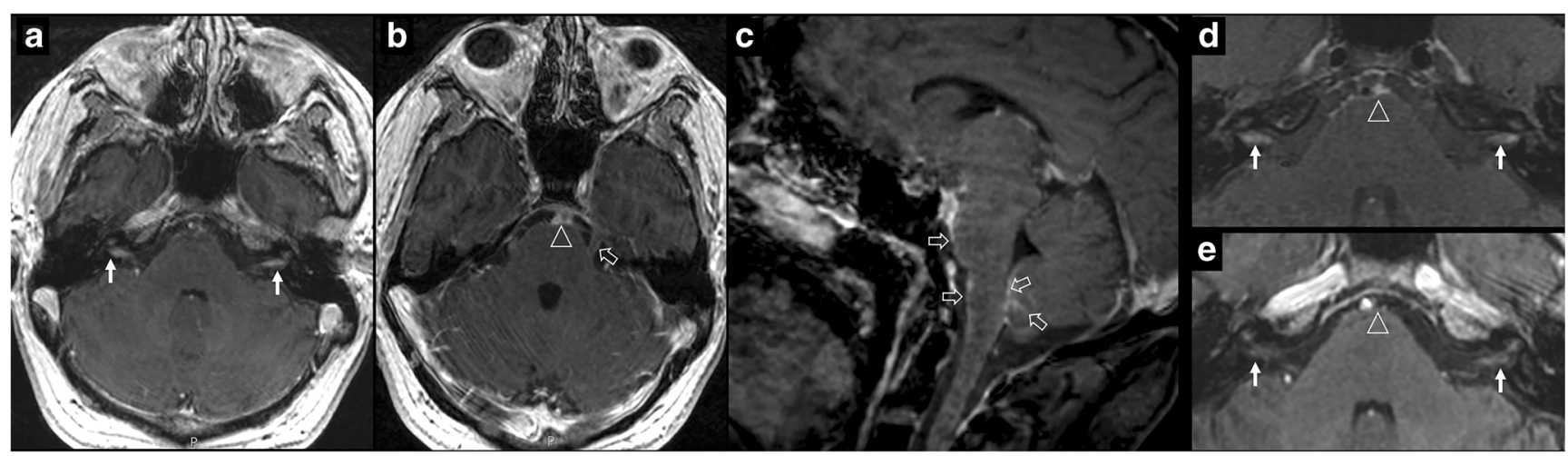

Fig. 2 Brain MRI imaging. a-c Axial and sagittal-enhanced T1-weighted images which obtained pre-steroid demonstrate abnormal enhancement in the internal auditory canals bilaterally (small white arrows), abnormal enhancement in the prepontine cistern (arrowhead), and meningeal enhancement along the trigeminal nerves, anterior aspect of the pons, medulla and along the inferior cerebellum (open white arrows). d-e Decreased abnormal enhancement within the auditory canals (small white arrows) and in the prepontine cistern (arrowhead) after corticosteroid administration (e) compared to pre-treatment (d)

worsening clinical presentation despite a negative CSF fungal antigen and persistently negative cultures after treatment with antifungal agents and ventriculoperitoneal shunting (Table 1). The patient underwent rapid clinical improvement following corticosteroid therapy. Supportive of an inflammatory syndrome were elevations in CSF cytokines including IL-6 and soluble CD25 and compatible MRI findings that resolved over a year. Fungal recurrence risk was ameliorated by high-dose itraconazole therapy throughout the steroid taper.

Previously, Klein et al. reported a 20-year-old woman who presented with CNS histoplasmosis mistaken for a glioma. Despite amphotericin treatment and ventriculostomy, her hydrocephalus progressed. She was started on prednisone with clinical improvement. Prednisone was tapered over 1 month but her hydrocephalus progressed a month later despite itraconazole therapy [7]. The clinical similarity between these two cases and a recently defined post-infectious inflammatory response syndrome (PIIRS) in non-HIV-associated cryptococcal meningoencephalitis suggests that such an inflammatory syndrome may also exist in Histoplasma infection and may explain the response to corticosteroids in the previously reported case. Other infectious and autoimmune diseases were excluded in the present case although anti-N-methyl-daspartate receptor antibodies were not measured and are an important, though rare differential [8].

PIIRS is defined as clinical deterioration in the setting of appropriate antifungal treatment and microbiologic control of the underlying fungal infection [9]. In cryptococcal meningoencephalitis, PIIRS is thought to be associated with a highly activated intrathecal $\mathrm{T}$ cell population following effective antigen presentation of newly lysed fungal organisms [10]. Crypto-PIIRS is highly responsive to initial pulses of antiinflammatory corticosteroid doses and may require extended tapers of corticosteroids to control flares of inflammation months after infection [9, 11, 12]. The clinical and laboratory similarities of these cases to Crypto-PIIRS postulate the
The refractory nature of this patient's neurological symptoms was attributed to an inflammatory syndrome based on a 
existence of a similar immune response in HIV-negative, previously healthy patients with CNS Histoplasma infection with elevations in IL-6 and sCD25. Shared also were a lack of TNF- $\alpha$ production, which may explain the lack of high fever in both patient populations. However, the present case did not show elevated levels of IL-10 as described in Crypto-PIIRS, associated with an alternatively activated M2 macrophage population [9], suggesting possible preservation of classical macrophage activation in the present case.

Th1 responses, consisting of pro-inflammatory cytokines, are integral to the host response to Histoplasma infection [13]. Dysregulated pro-inflammatory responses can also cause host damage. Although CSF cytokines were not assayed prior to corticosteroid therapy, we noted abnormally elevated proinflammatory cytokines (soluble CD25 or IL-2 receptor, IL-6, and IL-8) during the 2 days subsequent to steroid initiation that declined during corticosteroid therapy (Fig. 1). Soluble CD25 reflects immune activation and is used in the diagnosis of histoplasmosis-associated hemophagocytic lymphohistiocytosis [14]. IL-6 is involved in naïve T cell polarization, classically Th17 differentiation, and is released by activated astrocytes during CNS inflammation [15]. However, IL-17 was not detectable, similar to that often found in Crypto-PIIRS, which may be due to an overall Th1 bias in these syndromes [16]. IL-8 is another biomarker of neuroinflammation, primarily attracting and activating neutrophils and basophils [17]. Notably, the antiinflammatory cytokines IL-4 and IL-10, which suppress the generation of Th1 responses in histoplasmosis [18], were not elevated in the CSF of this patient. Similar inflammatory cytokine profiles, and a lack of anti-inflammatory cytokines, are seen in Crypto-PIIRS [11]. While we were not able to evaluate cellular markers of CSF inflammation due to the patient declining to come to the NIH, the finding of an elevated Il-6 level in the setting of an improved CRP on antifungal therapy, from an initial low value, suggests that CSF parameters were less likely due to systemic-CSF transmigration, similar to the intrathecal compartmentalization of inflammation in Crypto-PIIRS [11]. Previously, elevated blood CRP levels have been associated with elevations in systemic IL-6 due to induction of hepatic CRP synthesis by IL-6 [19]. MRI findings also reflected CNS inflammatory changes with abnormal enhancement in the prepontine cistern, as well as meningeal enhancement along the trigeminal nerves, anterior aspect of the pons, medulla, and inferior cerebellum, both similar to Crypto-PIIRS [20]. As reflected by clinical improvement and reduction of pro-inflammatory cytokines and improvement in MRI findings, the deleterious inflammatory response was likely controlled by corticosteroid treatment, suggesting a role for these parameters as biomarkers of CNS Histo-PIIRS, in addition to standard microbiological markers.

It is not clear if concurrent HCV infection was involved in host susceptibility to histoplasmosis in this case. Chronic $\mathrm{HCV}$ is known to effect both innate and adaptive immunity, including impaired effector $\mathrm{T}$ cell and dendritic cell function
[21]. Both are considered necessary for adequate histoplasmosis control [22], and concurrent HCV and histoplasmosis have been reported but are rare, typically associated with granulomatous hepatitis [23-25] and is also associated rarely with cryptococcal disease [26]. Future studies of disseminated histoplasma disease including more detailed immune studies and whole-exome sequencing offer the promise to shed more light on this rare disease.

Complicating the diagnosis of CNS histoplasmosis is the limited sensitivity and specificity of current microbiological laboratory tests (Table 1). According to a recent multicenter study of CNS histoplasmosis by Wheat et al., fungal cultures had low sensitivity and could take up to 6 weeks to demonstrate growth. Serological testing by complement fixation (CF) or immunodiffusion was not as helpful as EIA based on inferior sensitivity patterns [2]. Although not used in this case, a combined approach of testing CSF antigen and antibody by EIA is considered the most sensitive detection method (98\% sensitivity, $90.8 \%$ specificity) to diagnose CNS histoplasmosis [27]. Our patient was initially diagnosed based on CSF IgG detection by EIA (Table 1). (1,3)- $\beta$-D-Glucan levels were negative in the CSF despite high levels in the serum. CNS histoplasmosis is also known to relapse, with an estimated incidence of $6 \%$ [2]. On representation, our suspicion for relapsed infection was low based on a combination of negative CSF and serum antigen by EIA (Table 1). These results, in the setting of worsening neurological status and elevated CSF inflammatory markers, support the diagnosis of HistoPIIRS rather than relapsed infection. Of note, the antigen may be falsely negative in CNS histoplasmosis [27].

This patient's hospital course was also complicated by recurrent arthritis of the knee. On initial presentation, the diagnosis was MSSA septic arthritis. Histoplasmosis was not thought to be a causal factor at that time. On readmission, a specific inflammatory or infectious cause could not be identified. Cause of improvement could not be determined because treatment included cefazolin and prednisone. Whether the arthritis was PIIRS or infectious is unclear.

In summary, the present case suggests that CNS histoplasmosis may be followed by a post-infectious inflammatory response syndrome with similarity to PIIRS in cryptococcal meningoencephalitis. It can be recognized in a patient with confirmed CNS histoplasmosis by three criteria: (1) worsening clinical condition despite standard antifungal therapy, (2) improving serologic or antigenic parameters, and (3) evidence of elevated immune activation in the CSF. When recognized, CNS Histo-PIIRS may respond to corticosteroid therapy followed by a slow taper, during which reduction of CSF inflammatory markers and MRI findings may help confirm suppression of an overactive inflammatory response and help diagnose immunological flairs during the steroid taper. Continued antifungal therapy is crucial to prevent relapse caused by the immunosuppressive effects of prednisone. 
Author Contributions OD, SA, and PRW conceived and wrote the original draft. $\mathrm{BH}$ and DA collected clinical and laboratory data. DH reviewed the imaging data. All authors contributed to the review and editing process.

Funding OD is supported by the NIH Medical Research Scholars Program, a public-private partnership supported jointly by the NIH and contributions to the Foundation for the NIH from the Doris Duke Charitable Foundation, Genentech, the American Association for Dental Research, the Colgate-Palmolive Company, and other private donors. PRW, SA, and DAH are supported by the NIH Intramural Research Program

\section{Compliance with Ethical Standards}

Conflict of Interest LJW is a medical director, and part owner of MiraVista Diagnostics, a company that offers some of the described tests (antigen and antibody testing) commercially.

\section{References}

1. Kauffman CA. Histoplasmosis: a clinical and laboratory update. Clin Microbiol Rev. 2007;20(1):115-32. https://doi.org/10.1128/ CMR.00027-06.

2. Wheat J, Myint T, Guo Y, Kemmer P, Hage C, Terry C, et al. Central nervous system histoplasmosis: multicenter retrospective study on clinical features, diagnostic approach and outcome of treatment. Medicine. 2018;97(13):e245. https://doi.org/10.1097/ MD.0000000000010245.

3. Melzani A, De Reynal De Saint Michel R, Ntab B, Djossou F, Epelboin L, Nacher M et al. Incidence and trends in immune reconstitution inflammatory syndrome associated with Histoplasma capsulatum among people living with HIV: a 20-year case series and literature review. Clin Infect Dis Off Publ Infect Dis Soc Am. 2019. https://doi.org/10.1093/cid/ciz247.

4. Wright T, Coruh B, Fredricks D, Kim N. Immune reconstitution inflammatory syndrome associated with disseminated histoplasmosis and TNF-alpha inhibition. Med Mycol Case Rep. 2019;23:62-4. https://doi.org/10.1016/j.mmcr.2018.12.008.

5. Myint T, Chow FC, Bloch KC, Raymond-Guillen L, Davis TE, Wright PW et al. Detection of (1,3)-beta-d-glucan in cerebrospinal fluid in histoplasma meningitis. Journal of clinical microbiology. 2018;56(10). https://doi.org/10.1128/JCM.00663-18.

6. Richer SM, Smedema ML, Durkin MM, Herman KM, Hage CA, Fuller D, et al. Improved diagnosis of acute pulmonary histoplasmosis by combining antigen and antibody detection. Clin Infect Dis Off Publ Infect Dis Soc Am. 2016;62(7):896-902. https://doi.org/ 10.1093/cid/ciw007.

7. Klein CJ, Dinapoli RP, Temesgen Z, Meyer FB. Central nervous system histoplasmosis mimicking a brain tumor: difficulties in diagnosis and treatment. Mayo Clin Proc. 1999;74(8):803-7. https:// doi.org/10.4065/74.8.803.

8. Shin YW, Lee ST, Park KI, Jung KH, Jung KY, Lee SK, et al. Treatment strategies for autoimmune encephalitis. Ther Adv Neurol Disord. 2018;11:1756285617722347. https://doi.org/10. $1177 / 1756285617722347$.

9. Williamson PR. Post-infectious inflammatory response syndrome (PIIRS): dissociation of T-cell-macrophage signaling in previously healthy individuals with cryptococcal fungal meningoencephalitis. Macrophage (Houst). 2015;2. 10.14800/Macrophage.1078.

10. Neal LM, Xing E, Xu J, Kolbe JL, Osterholzer JJ, Segal BM et al. CD4(+) T cells orchestrate lethal immune pathology despite fungal clearance during Cryptococcus neoformans meningoencephalitis. MBio. 2017;8(6). https://doi.org/10.1128/mBio.01415-17.

11. Panackal AA, Wuest SC, Lin YC, Wu T, Zhang N, Kosa P, et al. Paradoxical immune responses in non-HIV cryptococcal meningitis. PLoS Pathog. 2015;11(5):e1004884. https://doi.org/10.1371/ journal.ppat.1004884.

12. Elsegeiny W, Marr KA, Williamson PR. Immunology of cryptococcal infections: developing a rational approach to patient therapy. Front Immunol. 2018;9:651. https://doi.org/10.3389/fimmu.2018. 00651.

13. Kroetz DN, Deepe GS. The role of cytokines and chemokines in Histoplasma capsulatum infection. Cytokine. 2012;58(1):112-7. https://doi.org/10.1016/j.cyto.2011.07.430.

14. Jabr R, El Atrouni W, Male HJ, Hammoud KA. Histoplasmosisassociated hemophagocytic lymphohistiocytosis: a review of the literature. Can J Infect Dis Med Microbiol. 2019;2019:71073268. https://doi.org/10.1155/2019/7107326.

15. Van Wagoner NJ, Benveniste EN. Interleukin-6 expression and regulation in astrocytes. J Neuroimmunol. 1999;100(1-2):124-39. https://doi.org/10.1016/s0165-5728(99)00187-3.

16. Panackal AA, Marr KA, Williamson PR. Dexamethasone in cryptococcal meningitis. New Engl J Med. 2016;375(2):188. https://doi. org/10.1056/NEJMc1605205\#SA1.

17. Bielekova B, Komori M, Xu Q, Reich DS, Wu T. Cerebrospinal fluid IL-12p40, CXCL13 and IL-8 as a combinatorial biomarker of active intrathecal inflammation. PLoS One. 2012;7(11):e48370. https://doi.org/10.1371/journal.pone.0048370.

18. Peng JK, Lin JS, Kung JT, Finkelman FD, Wu-Hsieh BA. The combined effect of IL- 4 and IL-10 suppresses the generation of, but does not change the polarity of, type- $1 \mathrm{~T}$ cells in Histoplasma infection. Int Immunol. 2005;17(2):193-205. https://doi.org/10. 1093/intimm/dxh200

19. Bermudez EA, Rifai N, Buring J, Manson JE, Ridker PM. Interrelationships among circulating interleukin-6, C-reactive protein, and traditional cardiovascular risk factors in women. Arterioscler Thromb Vasc Biol. 2002;22(10):1668-73. https://doi. org/10.1161/01.atv.0000029781.31325.66.

20. Hammoud DA, Mahdi E, Panackal AA, Wakim P, Sheikh V, Sereti I, et al. Choroid plexitis and ependymitis by magnetic resonance imaging are biomarkers of neuronal damage and inflammation in HIV-negative cryptococcal meningoencephalitis. Sci Rep. 2017;7(1):9184. https://doi.org/10.1038/s41598-017-09694-0.

21. Brenndorfer ED, Sallberg M. Hepatitis C virus-mediated modulation of cellular immunity. Arch Immunol Ther Exp (Warsz). 2012;60(5):315-29. https://doi.org/10.1007/s00005-012-0184-z.

22. Horwath MC, Fecher RA, Deepe GS Jr. Histoplasma capsulatum, lung infection and immunity. Future Microbiol. 2015;10(6):96775. https://doi.org/10.2217/fmb.15.25.

23. Ramesh V, Narreddy S, Gowrishankar S, Barigala R, Nanda S. A challenging case of pyrexia of unknown origin: adrenal histoplasmosis mimicking tuberculosis in a patient with chronic hepatitis $\mathrm{C}$. Trop Doct. 2018;49475518819622. https://doi.org/10.1177/ 0049475518819622

24. Rodriguez-Waitkus PM, Bayat V, George E, Sule N. Gastrointestinal histoplasmosis in a hepatitis C-infected individual. Mycopathologia. 2013;176(1-2):161-4. https://doi.org/10.1007/ s11046-013-9669-5.

25. Wang Z, Duarte AG, Schnadig VJ. Fatal reactive hemophagocytosis related to disseminated histoplasmosis with endocarditis: an unusual case diagnosed at autopsy. South Med J. 2007;100(2):208-11. https://doi.org/10.1097/SMJ. 0b013e31802b2812.

26. Pyrgos V, Seitz AE, Steiner CA, Prevots DR, Williamson PR. Epidemiology of cryptococcal meningitis in the US: 1997-2009. PLoS One. 2013;8(2):e56269. https://doi.org/10.1371/journal. pone.0056269. 
27. Bloch KC, Myint T, Raymond-Guillen L, Hage CA, Davis TE, Wright PW, et al. Improvement in diagnosis of histoplasma meningitis by combined testing for histoplasma antigen and immunoglobulin $\mathrm{G}$ and immunoglobulin $\mathrm{M}$ anti-histoplasma antibody in cerebrospinal fluid. Clin Infect Dis. 2018;66(1):89-94. https://doi. org/10.1093/cid/cix706.
Figures were created in GraphPad Prism.

Publisher's Note Springer Nature remains neutral with regard to jurisdictional claims in published maps and institutional affiliations. 